\title{
Acompanhamento farmacoterapêutico em pacientes com doença renal crônica
}

\author{
Pharmacotherapeutic follow-up in patients with chronic kidney disease \\ Stella Lucchesi ${ }^{\ominus}$, Danyelle Marini ${ }^{\ominus}$,Gabriel Carvalho* ${ }^{\circ}$ \\ Centro Universitário das Faculdades Associadas de Ensino, São João da Boa Vista, São Paulo, Brasil. *Autor para correspondência. E- \\ mail: carvallhovgs@gmail.com
}

Resumo: Fundamentos: Profissionais de saúde, nas últimas décadas, têm direcionado maior atenção as doenças crônicas, uma vez que são essas doenças as responsáveis por uma parcela significativa na morbidade da população mundial, atingindo desde jovens até os mais idosos. Atualmente os principais tratamentos disponíveis para doenças renais terminais são: diálise peritoneal automatizada, hemodiálise, diálise peritoneal ambulatorial contínua, diálise peritoneal intermitente e o transplante renal. Tais tratamento exercem parcialmente a função renal, aliviando sintomas da doença e preservando a vida do paciente, sem gerar a cura. Doentes renais crônicos fazem uso concomitante de vários medicamentos que, associados a alterações na taxa de filtração glomerular, múltiplas comorbidades e idade, aumentam o risco de interações medicamentosas. Objetivo: O objetivo do presente estudo é o acompanhamento farmacoterapêutico de pacientes com doença renal crônica visando a melhoria na sua qualidade de vida nesta população que, devido a prática da polifarmácia e a presença de diversas enfermidades, costuma ter sua qualidade de vida reduzida. Métodos: Dados presentes no prontuário do paciente e o SOAP (Subjective, Objective, Assessment, Plan), permitiu a coleta e organização de dados do paciente, a identificação dos problemas relacionados à farmacoterapia, a elaboração de um plano de cuidado em conjunto com o paciente e pôr fim a realização de um seguimento individual do paciente. Resultados: Dos 5 pacientes entrevistados, em 1 não ocorreu nenhuma interação medicamentosa, porém, 4 apresentaram interações medicamentosas, referente ao seu tratamento farmacológico totalizando 12 interações, sendo 6 (50\%) interações graves, 5 $(41,67 \%)$ moderadas e $1(8,33 \%)$ leves. Conclusões: Essa pesquisa proporcionou realizar o acompanhamento farmacoterapêutico em pacientes renais crônicos, que devido a sua condição possuem restrições de prescrições de diversas classes terapêuticas que podem gerar efeitos adversos em relação a função renal e idade o que resulta em polifarmácia e PRM's.

Palavras-chaves: doença renais, farmacêutico, interações.

\begin{abstract}
Background: Health professionals, in recent decades, have directed greater attention to chronic diseases, since these diseases are responsible for a significant portion of the morbidity of the world population, affecting from young people to the elderly. Currently, the main treatments available for endstage renal disease are: automated peritoneal dialysis, hemodialysis, continuous ambulatory peritoneal dialysis, intermittent peritoneal dialysis and kidney transplantation. Such treatments partially exert the renal function, alleviating disease symptoms and preserving the patient's life, without generating a cure. Chronic kidney patients use several drugs concomitantly, which, associated with changes in the glomerular filtration rate, multiple comorbidities and age, increase the risk of drug interactions. Objective: The aim of the present study is the pharmacotherapeutic follow-up of patients with chronic kidney disease, aiming at improving their quality of life in this population, which, due to the practice of polypharmacy and the presence of various diseases, usually has their quality of life reduced. Methods: Data present in the patient's medical record and the SOAP (Subjective, Objective, Assessment, Plan) allowed the collection and organization of patient data, the identification of problems related to pharmacotherapy, the elaboration of a care plan together with the patient and put an end to individual follow-up of the patient. Results: Of the 5 patients interviewed, in 1 there was no drug interaction, however, 4 had drug interactions, referring to their pharmacological treatment, totaling 12 interactions, with 6 (50\%) severe interactions, 5 (41.67\%) moderate and 1 ( $8.33 \%)$ light. Conclusions: This research allowed to carry out pharmacotherapeutic monitoring in chronic kidney patients, who, due to their condition, have prescription restrictions of different therapeutic classes that can generate adverse effects in relation to renal function and age, resulting in polypharmacy and DRPs.
\end{abstract}

Keywords: kidney disease, pharmacist, interactions. 


\section{Introdução}

Profissionais de saúde, nas últimas décadas, têm direcionado maior atenção as doenças crônicas, uma vez que são essas doenças as responsáveis por parcela significativa na morbidade da população mundial, atingindo desde jovens até os mais idosos. Destaca-se dentre essas doenças à insuficiência renal crônica (IRC) (Martins et al., 1996).

Caracterizada como lesão renal e dano irreversível e progressivo da função renal, a doença renal crônica pode ser dividida em seis estágios, os quais possuem como critério de divisão o grau da função renal. Desses seis estágios, caracteriza-se como estágio zero aquele no qual o grupo de risco apresenta ausência de lesão renal e estágio mais avançado (estágio 5) aquele no qual o grupo de risco apresenta doença renal crônica terminal, fase na qual o paciente apresenta sintomas intensos e as opções terapêuticas são baseadas em terapia renal substitutiva ou transplante renal (Martins et al., 1996).

A taxa de filtração glomerular em pacientes estágio 5 é inferir a $15 \mathrm{~mL} / \mathrm{min} / 1,72 \mathrm{~m}^{2}$, determinada via depuração de creatinina (Mortari et al., 2010). Pacientes em estágio 5 possuem a denominada falência funcional renal que representa o estágio mais avançado de perda da função progressiva renal com diagnóstico de doença renal crônica. (Bastos et al., 2010)

A partir de 2002 uma nova definição de doença renal crônica definiu que o estágio da doença não depende da sua causa, evidenciando frequência da doença e associação a alta taxas de morbimortalidade (Bastos et al., 2010).

Essenciais para a manutenção da homeostase do corpo humano, a diminuição progressiva da função renal compromete todos os outros órgãos. Avalia-se função renal a partir da filtração glomerular (FG) a qual diminui na Doença Renal Crônica (DRC) e quando associada a perda das funções endócrinas, regulatórias e excretórias do rim (Bastos et al., 2010). Assim considera-se a doença renal crônica como um problema de saúde pública, uma vez que além de contribuir para elevação das taxas de morbidade e mortalidade de uma população, essa doença afeta negativamente a qualidade de vida do paciente (Martins et al., 1996). Atualmente os principais tratamentos disponíveis para doenças renais terminais são: diálise peritoneal automatizada (DPA), hemodiálise (HD), diálise peritoneal ambulatorial contínua (DPAC), diálise peritoneal intermitente (DPI) e o transplante renal (TX). Tais tratamento exercem parcialmente a função renal, aliviando sintomas da doença e preservando a vida do paciente, sem gerar a cura (Thomé, 1996)

Análises epidemiológicas recentes demonstram cerca de um milhão de pessoas em todo o mundo que são portadoras de doença renal crônica terminal (DRCT) e se submetem à terapia de substituição renal (TSR), número que cresce com o passar dos anos. O crescente número deve-se ao aumento no número de casos de diabetes mellitus, aumento da expectativa de vida e a hipertensão arterial (Martins et al., 1996) O controle do diabetes mellitus e da hipertensão arterial é feito a partir da utilização de fármacos seguros e eficazes (Martins et al., 1996)

Avanços terapêuticos e tecnológicos em dialise e toda sua área favorecem a crescente sobrevida dos pacientes renais crônico, porém não assegura o retorno à vida em aspectos qualitativos. Pacientes dependentes de tecnologia avançada para sobreviver possuem limitações cotidianas, mudanças psicossociais e alterações na qualidade de vida, muitas vezes com perda do emprego, dieta restritiva, alterações em sua imagem corporal (Madeira et al., 1998)

Buscando melhorar a qualidade de vida desses pacientes, tem se voltado atenção para a terapêutica renal, a qual baseia-se no bem-estar físico e mental que resulta em autonomia do paciente, retorno a atividades de lazer e trabalho, retomada da esperança e utilidade (Law, 2002)

O tratamento hemodiálico corresponde a inserção de monotonia e restrição ao dia a dia do paciente renal crônico, que favorece o sedentarismo e torna necessário suporte formal em atenção à saúde, ou seja, dependência da equipe de saúde, máquina e suporte informal para garantia de vida e cuidado necessário (Ribeiro, 2000).

Saber reconhecer os aspectos que englobam a doença de base, o estágio da doença, possíveis complicações correspondem ao tratamento dos pacientes com doença renal crônica (Bastos et al., 2010). Doentes renais crônicos fazem uso concomitante de vários medicamentos que, associados a alterações na taxa de filtração glomerular, múltiplas comorbidades e idade, aumentam o risco de interações medicamentosas (IM). Definese interação medicamentosa aquela que é responsável por resposta farmacológica ou clinica diferente da esperada após administração de medicamentos combinados, gerando reduzida eficiente ou elevada toxicidade (Marquito, 2014)

Classificam-se interações medicamentosas como interações medicamentosas graves, representadas por interações que podem causar a morte ou sequelas irreversíveis a saúde do paciente; interações moderadas representadas por interações que causam a piora do quadro clinico do paciente, necessitando-se de 
hospitalização do paciente ou aumento do tempo de hospitalização; interações leves representadas por interações que causam pequeno incomodo ou são assintomáticas, não afetando a qualidade da terapia e não necessitando de tratamento (Sehn, 2003).

Interações farmacêuticas, farmacocinéticas e farmacodinâmica são mecanismos utilizados para classificar as interações medicamentosas. As interações farmacêuticas antes e durante a administração de medicamentos, onde ocorrem interações físico químicas entre dois ou mais medicamentos, quando estes são misturados em uma mesma solução ou recipiente e essa mistura faz com que um dos medicamentos ou os dois perca seu efeito terapêutico, devido a incompatibilidade dos medicamentos misturados ou incompatibilidade do veículo ao qual foram misturados, podendo ser observada, a precipitação ou turvação de uma solução, mudança de cor do medicamento ou inatividade do princípio ativo (Secoli, 2001)

Introduzida nos moldes assistenciais de saúde a Atenção Farmacêutica é importante para a prática mundial farmacêutica. Assim, define-se como acompanhamento farmacoterapêutico a prática da assistência farmacêutica, na qual o farmacêutico detecta, previne e busca solucionar Problemas Relacionados a Medicamentos (PRM) por meio de um acompanhamento sistematizado e documentado por determinado período de tempo, onde o paciente coopera com equipe multidisciplinar que oferece cuidados a esse paciente, visando alcançar melhora da qualidade de vida do paciente (Lima et al., 2016).

$\mathrm{Na}$ atenção farmacêutica há o serviço voltado para o paciente, sendo denominado como acompanhamento farmacoterapêutico, o qual consiste em vários encontros com o paciente onde identificam-se problemas relacionados a medicamentos e resultados negativos da farmacoterapia utilizada, é realizada analise de suas causas e as intervenções realizadas são documentadas, visando resolver os problemas medicamentosos ou preveni-los. Assim, no acompanhamento farmacoterapêutico há elaboração conjunta de um plano de cuidado do paciente e continuidade do cuidado provido pelo farmacêutico (PSA, 2011; OPAS, 2002;)

O objetivo do presente estudo é o acompanhamento farmacoterapêutico de pacientes com doença renal crônica visando a melhoria na sua qualidade de vida nesta população que, devido á pratica da polifarmácia e a presença de diversas enfermidades, costuma ter sua qualidade de vida reduzida.

\section{Material e métodos}

A coleta de informações foi realizada utilizando de dados presentes no prontuário dos paciente, bem como da utilização de um método de registro denominado SOAP (Subjective, Objective, Assessment, Plan), este permitiu a coleta e organização de dados do paciente, a identificação dos problemas relacionados à farmacoterapia, a elaboração de um plano de cuidado em conjunto com o paciente e pôr fim a realização de um seguimento individual do paciente, o qual permitirá verificar se ocorreu o alcance das metas terapêuticas. Os indivíduos que fizeram parte do estudo realizam tratamento no Instituto de Doenças Renais (IDR) localizado no município de São João da Boa Vista e são portadores de Doença Renal Crônica. Os problemas encontrados relacionados ao tratamento foram explanados ao médico prescritor, com intuito de alcançar uma farmacoterapia eficaz.

\section{Resultados e discussão}

A pesquisa abordou cinco pacientes que realizavam tratamento no Instituto Renal de Doenças Crônicas (IDR) de São João da Boa Vista - SP. Estes pacientes eram compostos por mulheres (40\%) homens (60\%).

Referente ao grau de escolaridade verificou que o maior percentual de escolaridade foi dos homens, sendo ao ensino fundamental (80\%) e ensino técnico (10\%). Já para os homens, todos os pacientes relataram que somente tinham o ensino fundamental (100\%) (Tabela 1).

No que se refere a faixa etária, 3 pacientes possuíam suas idades abaixo de 60 anos e 2 pacientes suas idades acima de 60 anos, caracterizando idosos. Segundo a OMS (2015) existem diferentes fases da velhice sendo idade madura dos 60 aos 69 anos (20\%), idade avançada dos 70 aos 89 (00\%) e grande idade acima dos 90 (00\%) (Tabela 2).

Ao iniciar as consultas, os 5 pacientes foram questionados sobre a Qualidade de Vida e a Percepção Geral da Saúde, em que eles se auto avaliaram. Dos 5 pacientes, 2 deram a nota 10, relatando que são felizes e se sentem muito bem, apesar de suas patologias as quais não interferem em sua qualidade de vida, 1 deu a nota 8 , justificando que apesar de sua patologia, sente-se bem, mas as vezes não consegue controlar os sintomas, 1 deu a nota 7 , justificando que as patologias interferem em sua plena qualidade de vida. Já 1 relatou nota 6 , justificando que sua doença vascular o atrapalha tanto na locomoção quanto as dores frequentes e, infelizmente, não consegue evolução nem melhora do tratamento. 
Tabela 1. Distribuição de entrevistados segundo a escolaridade.

\begin{tabular}{|c|c|c|c|c|c|c|}
\hline \multirow[t]{2}{*}{ Nível De Escolaridade } & \multicolumn{2}{|c|}{ Homens } & \multicolumn{2}{|c|}{ Mulheres } & \multicolumn{2}{|c|}{ Total } \\
\hline & $\mathbf{N}$ & $\%$ & $\mathbf{n}$ & $\%$ & $\mathbf{n}$ & $\%$ \\
\hline Técnico Completo & - & - & 1 & 50 & 1 & 20 \\
\hline Fundamental Completo & - & - & 1 & 50 & 1 & 20 \\
\hline Fundamental Incompleto & 3 & 100 & - & - & 3 & 60 \\
\hline Total & 3 & 100 & 2 & 100 & 5 & 100 \\
\hline
\end{tabular}

Tabela 2. Distribuição de entrevistados segundo a faixa etária.

\begin{tabular}{lcccccc}
\hline Faixa Etária & \multicolumn{2}{c}{ Homens } & \multicolumn{2}{c}{ Mulheres } & \multicolumn{2}{c}{ Total } \\
\cline { 2 - 7 } & $\mathrm{N}$ & $\%$ & $\mathbf{n}$ & $\mathbf{n}$ & $\%$ \\
$35-40$ & - & - & 2 & 100 & 2 & 40 \\
$40-45$ & - & - & - & - & - \\
$46-50$ & - & - & - & - & - \\
$56-60$ & 2 & 40 & - & - & 4 & 40 \\
$61-65$ & 1 & 20 & - & 100 & 5 & 100 \\
Total & 3 & 100 & 2 & & & \\
\hline
\end{tabular}

Dos 5 pacientes entrevistados, as patologias apresentadas foram diabetes mellitus 1 (20\%), problemas vasculares 2 (40\%), Esporão do Calcâneo 1 (20\%) e hipertensão 5 (100\%). Todos os pacientes passam pelo processo de hemodiálise (Figura 1).

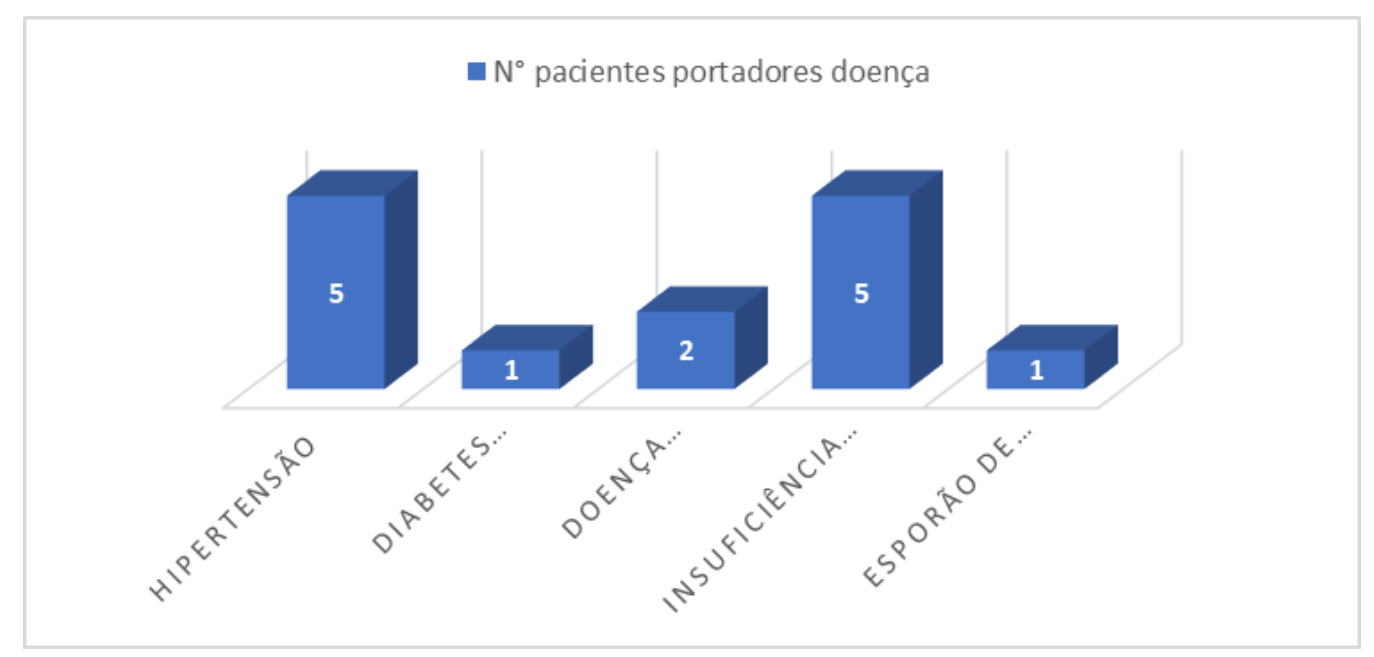

Figura 1. Distribuição de patologias segundo a quantidade de pacientes que apresentam cada. Fonte: Autores, 2019.

Atendidos no IDR do bairro Parque Colina da Mantiqueira e os medicamentos são adquiridos, em sua maioria, em unidades de saúde e nas redes de farmácia popular. O acompanhamento farmacoterapêutico foi realizado na sala de espera do IDR, em dias de sessões de hemodiálise. A tabela 4 relaciona os medicamentos prescritos aos pacientes, organizando-os pela classificação ATC e os medicamentos mais utilizados foram ácido acetilsalíco 4 (13,79\%), Losartana 4 (13,79\%) (Tabela 3).

Dos 5 pacientes entrevistados, em 1 não ocorreu nenhuma interação medicamentosa, porém, 4 apresentaram interações medicamentosas, referente ao seu tratamento farmacológico totalizando 12 interações, sendo 6 (50\%) interações graves, 5 (41,67\%) moderadas e 1 (8,33\%) leves.

No que se refere a interações graves, são aquelas que podem trazer piora no quadro de vida do paciente, necessitando de intervenções no tratamento. Das 12 interações medicamentosas encontradas 6 (50\%) delas foram graves. Vale ressaltar que três dessas interações ocorreram mais de uma vez, sendo cada uma localizada em um paciente distinto do outro (Tabela 4). 
Tabela 3. Medicamentos prescritos de acordo com a classificação ATC.

\begin{tabular}{lccc}
\hline ATC & Medicamento & $\mathbf{n}$ & $\mathbf{\%}$ \\
A02BC01 & Omeprazol cápsula 20 mg & 3 & 10,34 \\
B01AC06 & Ácido acetilsalicílico comprimido $100 \mathrm{mg}$ & 4 & 13,79 \\
B01AC23 & Cilostazol comprimido 50 mg & 2 & 6,9 \\
C03CA01 & Furosemida comprimido 40 mg & 3 & 10,34 \\
C07AB03 & Atenolol comprimido 50 mg & 3 & 10,34 \\
C08CA01 & Anlodipino besilato comprimido 5 mg & 2 & 6,9 \\
C09CA01 & Losartana comprimido 50 mg & 4 & 13,79 \\
C10AA01 & Sinvastatina comprimido revestido 10 mg & 2 & 6,9 \\
C07AG02 & Carvedilol comprimido 6,25 mg & 1 & 3,45 \\
A11DA03 & Benfotiamina comprimido 150 mg & 1 & 3,45 \\
A10AE & Insulina NPH 100 & 1 & 3,45 \\
C08CA05 & Nifedipina comprimido 20 mg & 1 & 3,45 \\
C02LG01 & Apresolina 25mg & 1 & 3,45 \\
R06AB02 & & 1 & 3,45 \\
Total & Dexclorfeniramina comprimido 2 mg & $\mathbf{2 9}$ \\
\hline
\end{tabular}

Tabela 4. Distribuição dos pacientes segundo a presença de interações graves nas prescrições.

\begin{tabular}{lccc}
\hline Medicamentos & n & \% & Consequência Da Interação \\
\hline Ácido acetilsalicílico e Cilostazol & 2 & 33,33 & $\begin{array}{c}\text { Pode resultar em aumento do risco de sangramento (isto é, } \\
\text { hemorragias). }\end{array}$ \\
$\begin{array}{l}\text { Ácido acetilsalicílico e Furosemida } \\
\begin{array}{l}\text { Cilostasol e Omeprazol } \\
\text { TOTAL }\end{array}\end{array}$ & 3 & 50 & $\begin{array}{c}\text { Pode resultar em aumento do risco de toxicidade renal. } \\
\text { Pode aumentar a atividade do Cilostazol, ou seja, efeito } \\
\text { vasodilatador e efeito anti agregante plaquetário }\end{array}$ \\
\hline
\end{tabular}

Interações moderadas podem resultar em agravamento do problema de saúde do paciente, assim requerendo uma alteração no tratamento. Das prescrições analisadas, ocorreram $5(41,67 \%)$ de interações moderadas, das quais 3 ocorreram mais de uma vez (Tabela 5).

Tabela 5. Distribuição dos pacientes segundo a presença de interações moderadas nas prescrições.

\begin{tabular}{|c|c|c|c|}
\hline Medicamentos & $\mathbf{N}$ & $\%$ & Consequência da Interação \\
\hline Atenolol e Insulina NPH & 1 & 20 & $\begin{array}{l}\text { Pode resultar em hipoglicemia ou hiperglicemia com } \\
\text { diminuição dos sintomas da hipoglicemia. }\end{array}$ \\
\hline Ácido acetilsalicílico e Insulina NPH & 1 & 20 & Pode resultar em aumento do risco de hipoglicemia. \\
\hline Atenolol e Ácido acetilsalicílico & 3 & 60 & $\begin{array}{l}\text { Pode resultar diminuição na produção de protaglandinas } \\
\text { renais e aumento da pressão sanguinea. }\end{array}$ \\
\hline TOTAL & 5 & $100 \%$ & 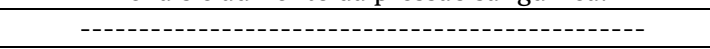 \\
\hline
\end{tabular}

As interações leves são acometidas por efeitos clínicos mais escassos com manifestações que aumentam ou não os efeitos colaterais. Não são realizadas alterações no tratamento do paciente nesse caso. A interação entre apresolina e furosemida foi a única encontrada (Tabela 6).

Tabela 6. Distribuição dos pacientes segundo a presença de interações leves nas prescrições.

\begin{tabular}{lccc}
\hline MEDICAMENTOS & N & \% & CONSEQUÊNCIA DA INTERAÇÃO \\
\hline Apresolina e Furosemida & 1 & 100 & $\begin{array}{c}\text { Pode resultar em aumento da resposta diurética da } \\
\text { furosemida. }\end{array}$ \\
TOTAL & & $100 \%$ & ---------------------------------- \\
\hline
\end{tabular}

A Sociedade de Geriatria Americana (AGS, 2015) por mieo dos Critérios de Beers, indica que medicamentos listados como inadequados para os idosos tendem aumentar e expor em risco a saúde dos idosos. Garantir a escolha de um medicamento considerado como adequado é primordial para a prevenção de eventos adversos. Nas prescrições analisadas, verificou-se o uso de 10 medicamentos classificados inadequados, segundo este critério. O medicamento inadequado mais prescrito foi o omeprazol (30\%), dos 10 pacientes atendidos 6 faz o uso (Tabela 7). 
Tabela 7. Distribuição dos medicamentos inapropriados segundo o critério de Beers.

\begin{tabular}{|c|c|c|c|c|c|c|}
\hline Medicamento & ATC & $\mathbf{n}$ & $\%$ & Racionalidade & $\begin{array}{l}\text { Qualidade da } \\
\text { Evidência }\end{array}$ & $\begin{array}{c}\text { Força da } \\
\text { Recomenda } \\
\text { ção } \\
\end{array}$ \\
\hline Furosemida & C & 1 & 14 & $\begin{array}{c}\text { Risco de hipovolemia e } \\
\text { desidratação com tendência ao } \\
\text { desenvolvimento de tromboses }\end{array}$ & Moderado & Forte \\
\hline Cilostasol & C & 1 & 14 & $\begin{array}{l}\text { Potencial para aspiração e efeitos } \\
\text { adversos. }\end{array}$ & Moderado & Forte \\
\hline Omeprazol & A & 1 & 14 & $\begin{array}{l}\text { Risco de infecções por Clostridium } \\
\text { difficile e perda e fraturas ósseas. }\end{array}$ & Alto & Forte \\
\hline Ácido Acetilsalicílico & B & 2 & 30 & $\begin{array}{l}\text { Risco de sangramento grave. Usar } \\
\text { com cautela para adultos com } \\
\text { idade maior ou igual a } 70 \text { anos. }\end{array}$ & Moderado & Forte \\
\hline Anlodipino & C & 1 & 14 & $\begin{array}{l}\text { Risco potencial de hipertensão e } \\
\text { isquemia do miocárdio }\end{array}$ & Alto & Forte \\
\hline Insulina NPH & B & 1 & 14 & $\begin{array}{l}\text { Risco de hipoglicemia, sem } \\
\text { melhora controle da } \\
\text { hiperglicemia. }\end{array}$ & Moderado & Forte \\
\hline
\end{tabular}

Observação: $\mathrm{A}$ = medicamentos que atuam no aparelho digestivo e metabolismo; $\mathrm{B}=$ medicamentos que atuam no sangue e órgãos hematopoiéticos; $\mathrm{N}$ = medicamentos que atuam no sistema nervoso; $\mathrm{C}=$ medicamentos que atuam no sistema cardiovascular; $\mathrm{R}=$ medicamentos que atuam no sistema respiratório.

Resultados de problemas relacionados à utilização dos medicamentos (PRMs), os efeitos indesejáveis podem influenciar de modo negativo na farmacoterapia do paciente. De tal modo, dos 5 pacientes analisados 4 apresentaram PRM, dos quais 2 foram PRM de insegurança (sendo 7 por medicamentos inadequados de acordo com os Critérios de Beers, 12 por ser interação medicamentosa, os quais acarretam danos a terapia) (Tabela 8).

Tabela 8. Classificação dos problemas relacionados aos medicamentos.

\begin{tabular}{lccc}
\hline PRM & Tipo & $\mathbf{n}$ & \% \\
\hline Necessidade & Problema de saúde não tratado & 0 & 0 \\
Insegurança & Medicamentos contraindicados & 7 & 36,84 \\
& Interação medicamentosa & 12 & 63,16 \\
\hline TOTAL & & 19 & 100 \\
\hline
\end{tabular}

Devido as interações medicamentosas e os problemas relacionados aos medicamentos encontrados, se fez necessário realizar intervenções em conjunto com a equipe multidisciplinar em Saúde no qual o paciente em estudo faz tratamentos terapêuticos.

\section{Considerações finais}

Essa pesquisa proporcionou realizar o acompanhamento farmacoterapêutico em pacientes renais crônicos, que devido a sua condição possuem restrições de prescrições de diversas classes terapêuticas que podem gerar efeitos adversos em relação a função renal e idade o que resulta em polifarmácia e PRM's. O farmacêutico atua visando estabelecer critérios para elaborar o perfil farmacoterapêutico e a terapêutica farmacológica adequada para o acompanhamento de seus pacientes. Pacientes Renais Crônicos se encaixam nesse perfil, sendo o acompanhamento farmacoterapêutico de grande importância uma vez que possibilita ao paciente maior proximidade do farmacêutico, que pode pôr em pratica os conhecimentos adquiridos durante a graduação, focando no paciente e na melhora de sua qualidade de vida. Aos pacientes que precisaram de intervenção medicamentosa, foi realizada reunião com o médico prescritor, na qual foi explanada cada interação e uma possível solução do problema encontrado.

Constata-se a ocorrência de PRM's nos pacientes, e a necessidade da atuação do farmacêutico nesse seguimento para aprimorar a farmacoterapia, manter a segurança do paciente e assegurar o uso racional de medicamentos. 


\section{Referência}

American Geriatrics Society [AGS]. 2015. Updated Beers Criteria for Potentially Inappropriate Medication Use in Older Adults. Jornal of the American Geriatrics Society, 63(11), 2227-2246.

Bastos, M. G., Bregman, R. \& Kirsztajn, G. M. 2010. Doença renal crônica: frequente e grave, mas também prevenível e tratável. Revista da Associação Médica Brasileira, 56(2), 248-253, 2010.

Law, M. 2002. Participation in the occupations everyday life. The American Journal of Occupational Therapy, 56(6), 640-649.

Lima, T. A. M., Fazan, E. R., Pereira, L. L. V., \& de Godoy, M. F. 2016. Acompanhamento farmacoterapêutico em idosos. Arquivos de Ciências da Saúde, 23(1), 52-57.

Madeira, E. P. Q., Lopes, G. S., \& Santos, S. F. F. 1998. A investigação epidemiológica na prevenção da insuficiência renal terminal. Ênfase no estudo da agregação familiar. Medonline, 1(2).

Marquito, A. B., Fernandes, N. M. D. S., Colugnati, F. A. B., \& Paula, R. B. D. 2014. Identifying potential drug interactions in chronic kidney disease patients. Brazilian Journal of Nephrology, 36, 26-34.

Martins, L. M., França, A. P. D., \& Kimura, M. 1996. Qualidade de vida de pessoas com doença crônica. Revista Latino-Americana de Enfermagem, 4, 5-18.

Mortari, D. M., Menta, M., Scapini, K. B., Rockembach, C. W. F., Duarte, A., \& Leguisamo, C. P. 2010. Qualidade de vida de indivíduos com doença renal crônica terminal submetidos à hemodiálise. Sci Med, 20(2), 156-60.

Organização Mundial da Saúde [OMS]. 2015. Relatório Mundial de Envelhecimento e Saúde-Resumo.

Organização Panamericana da Saúde [OPAS]. 2002. Consenso Brasileiro de Atenção Farmacêutica: proposta. Brasília, DF: OPAS.

Pharmaceutical Society of Australia [PSA]. 2011. Standard and guidelines for pharmacists performing clinical interventions. Sidney: PSA.

Ribeiro, R. C. H. M. 2000. A condição do idoso com insuficiência renal crônica. Dissertação de mestrado. Ribeirão Preto, SP: Escola de Enfermagem de Ribeirão Preto.

Sehn, R., Camargo, A. L., Heineck, I., \& Ferreira, M. B. C. 2003. Interações medicamentosas potenciais em prescrições de pacientes hospitalizados. Infarma, 15(9-10), 77-81.

\section{Minicurrículo}

Stella Lucchesi. Graduando em Farmácia pelo Centro Universitário das Faculdades Associadas de Ensino- FAE.

Danieli Marini. Doutora em Educação pela Universidade Metodista de Piracicaba (UNIMEP); Mestre em Biologia Celular e Molecular pelas Universidade Júlio Mesquita de São Paulo (UNESP); Especialista em Docência do Ensino Superior pela Gama Filho; Especialista em Cosmetologia pela UNIMEP; Graduada em Farmácia Bioquímica pela UNIMEP. Atua como docente e Coordenadora nas Faculdades Integradas Maria Imaculada; Docente do curso de graduação em Farmácia e do Mestrado do Centro Universitário das Faculdades Associadas de Ensino (UNIFAE), Presidente do Comitê de Ética do UNIFAE; Conselheira pelo Conselho Regional de Farmácia do Estado de São Paulo (CRF-SP); Membro do Comitê de Educação Permanente do CRFSP e da Comissão de Educação do CRF-SP.

Gabriel Carvalho. Graduando em Farmácia pelo Centro Universitário das Faculdades Associadas de Ensino- FAE.

Como citar: Lucchesi, S., Marini, D., \& Carvalho, G. G. 2021. Acompanhamento farmacoterapêutico em pacientes com doença renal crônica. Pubsaúde, 7, a232. DOI: https://dx.doi.org/10.31533/pubsaude7.a232

Recebido: 24 jul. 2021.

Revisado e aceito: 3 ago. 2021.

Conflito de interesse: os autores declaram, em relação aos produtos e companhias descritos nesse artigo, não ter interesses associativos, comerciais, de propriedade ou financeiros que representem conflito de interesse.

Licenciamento: Este artigo é publicado na modalidade Acesso Aberto sob a licença Creative Commons Atribuição 4.0 (CC-BY 4.0). 